\title{
What can music tell us about social interaction?
}

Alessandro D'Ausilio ${ }^{1}$, Giacomo Novembre ${ }^{2}$ Luciano Fadiga $^{1,3}$ \& Peter E. Keller ${ }^{2}$

1) IIT - Italian Institute of Technology, RBCS - Robotics, Brain and Cognitive Sciences, Genova, Italy

2) The MARCS Institute, University of Western Sydney, Australia

3) University of Ferrara, Section of Human Physiology, Ferrara, Italy

Corresponding author: Alessandro D’Ausilio, email: alessandro.dausilio@iit.it

\begin{abstract}
Humans are innately social creatures, but cognitive neuroscience, traditionally focused on individual brains, is just beginning to investigate social cognition through realistic interpersonal interaction. Music provides an ideal domain for doing so, as it offers a promising solution for balancing the trade-off between ecological validity and experimental control when testing cognitive and brain functions. Musical ensembles constitute a microcosm that provides a platform for parametrically modeling the complexity of human social interaction.
\end{abstract}

Published in:

Trends Cogn Sci 19(3):111-4.

doi: 10.1016/j.tics.2015.01.005

https://www.sciencedirect.com/science/article/abs/pii/S1364661315000066 


\section{Probing human interaction through music}

Human cognition and brain organization are shaped by the fact that we are innately social creatures. Cognitive neuroscience has nevertheless been slow to acknowledge that studying isolated individuals may be an ill-posed scientific approach, mainly because-when dealing with complex behaviors-experimental rigor is inversely related to ecological naturalness. Recently, however, a growing awareness of the profound impact of social context on neural functions has highlighted the need for experimental paradigms that enable to investigate the brain functions during real social interaction. We propose that music offers a promising solution for balancing the trade-off between experimental control and ecological naturalness in socialcognitive neuroscience.

Musical ensemble performance is a universal means of non-verbal communication that is achieved through specialized and codified forms of social interaction. Ensemble performers build on the human predisposition for musicality (observable already in infant-caregiver interactions) by undergoing years of training to hone sensorimotor, cognitive, and social skills that allow shared communicative goals to be developed and fulfilled through real-time interpersonal coordination.

Group-level musical coordination can be considered a microcosm of social interaction. Individual musicians function as processing units within a complex dynamical system (the ensemble) whose goal is to communicate musical meaning (which is aesthetic and affective in nature) to an audience. Information flows simultaneously to and from each unit, and the system as a whole relies upon predictive models and adaptive mechanisms to meet the real-time demands of interpersonal coordination. As in more general forms of social interaction, coperformers behave in complex but formalized (rule-based) ways that are constrained by the tools they use (musical instruments), conventions (genre-specific performance styles and leader-follower roles), and often a script (the musical score).

We argue that these musical constraints are beneficial from an experimental perspective (see Box 1) and may translate into ready-made experimental tasks, high levels of intrinsic motivation, and rich ecological settings. Research in the musical domain is therefore well placed to shed light upon topics of general interest for social 
cognition while balancing the trade-off between experimental control and ecological validity.

\section{Empirical study of music as social interaction}

The use of music as a model system for social interaction has inspired novel perspectives and original questions that touch on the core of human social cognition.

Musical ensemble performance is a form of social collaborative behavior that requires multiple individuals to anticipate and adapt to each other's actions. The operationalization of sensorimotor mechanisms that support such interpersonal coordination can reveal underling social dynamics between performing musicians, such as emerging leadership roles. A study of string quartets, for example, quantified the operation of mutual adaptive timing mechanisms between co-performers at the millisecond level and used this information to reveal how two quartets playing the same piece exhibit distinct leadership strategies characterized by first-violin-led autocracy versus democratic group organization [1].

Ensembles can be studied as complex, interactive systems in which information is transmitted through body movements and processed through the observation of their effects. Such sensorimotor information transfer (or flow) is representative of most non-verbal communicative behavior, but it is an elusive construct that can be difficult to control and quantify. In music, however, the score itself can be designed to regulate information flow between co-performers without introducing unnatural experimental requirements. Recent research has employed mathematical tools, such as Granger Causality, to quantify the information flow between musicians. One study found that increased information flow from conductor to musicians, together with decreased musician-to-musician coordination, was associated with musical experts' judgments of ensemble aesthetic quality [2]. Related analytical approaches have been used to study musical dominance and leadership [3] and to distinguish solo and ensemble modes of performance [4].

The encoding and decoding of sensorimotor information is mediated by the coupling of sensory and motor brain systems and a mirror-neuron matching mechanism that supports this function by motorically representing other's behaviour. A musical task 
requiring piano performance has identified distinguishable patterns of motor activity for self- vs. other-related neural processes during action co-representation. Pianists listened to a recording of a rehearsed left-hand part, while performing the complementary right-hand part of a musical piece. Corticospinal excitability associated with the resting left forearm increased when pianists were led to believe that they were interacting with a co-performer playing the complementary part behind a screen [5]. The function of such co-representation was addressed in a further study, which showed that repetitive transcranial magnetic stimulation ( $r T M S)$ over the right motor cortex impaired synchronization with tempo changes in the contralateral lefthand part when it had been trained beforehand [6]. The use of a musical task thus revealed that co-representation is an inherently social mechanism that facilitates the simulation and integration of one's own and others' actions during dynamic interaction.

The neurophysiological processes supporting collaborative performance in musical ensembles has extended beyond the individual brain to include the relationship between processes occurring within multiple interacting brains [7, 8]. The emerging field of hyperscanning permits the study of how interpersonal information flow results in coupled patterns of intra- and inter-brain dynamics. By capitalizing on the fact that ensemble musicians are relatively stationary during performance (which allows high signal-to-noise ratios when recording brain activity [9]), neuroscientists have been able to dissociate processes related to monitoring the self's performance and the joint action outcome [10] and to investigate the amount of inter-brain information flow. Work on the latter topic suggests that specific patterns of neural coupling (i.e. in-phase synchronization) between participants are associated with leader-follower dynamics [7] and that inter-brain system-wide neuronal oscillatory phase coherence is up-regulated during periods requiring higher coordination [8].

Music is generally an effective means by which to study cooperation and pro-social behaviour from a neural perspective. Recent research has shown that joint drumming increases activity in the caudate nucleus (a key area in the brain's reward system) and promotes prosocial behaviour in adults [11] and children [12]. Links between interpersonal synchronization and social-psychological factors - such as cooperativeness have been explored in studies involving sensorimotor synchronization with human 
and computer-controlled virtual partners. Interaction with cooperative virtual partners leads to the activation of cortical midline structures related to social-affective processes, whereas interaction with partners that make coordination difficult increases activation in lateral prefrontal areas associated with executive functions and cognitive control [13]. Virtual partner interaction has also proven informative about leaderfollower dispositions, showing that leaders (individuals who set the tempo) exhibit greater self-focus and stronger activation of agency-related brain regions than followers [14].

\section{A design taxonomy of musical ensemble interaction}

The research reviewed above falls into multiple taxonomic classes that traverse a continuum of ecological interaction. These classes include an individual interacting with a) a recording, b) a computer-controlled virtual partner that responds to the individual, c) another individual in a duo, d) multiple individuals in mixed ensembles (extending to large orchestras), and e) others in the presence of a live audience (see Figure 1).

This continuum allows hypotheses to be tested through systematic manipulations of musical interaction that are parametrically graded in terms of the degree of ecological naturalness and experimental control. As ecological validity increases, the directionality of the interaction becomes more complex: from unidirectional, to bidirectional or multidirectional, involving a computer, other musicians and also an audience. Increasing ecological validity offers the possibility to explore natural and unconstrained interaction, but it is also associated with higher uncertainty concerning others' actions, which implies decreasing experimental control (see Box 1). The taxonomy we propose may be used to guide the process of testing whether findings from tightly controlled contexts generalize to progressively richer ecological settings.

\section{Future directions and concluding remarks}

Musical ensembles enable the investigation of the neural and behavioral markers of nonverbal communication within a broad spectrum of social interactive contexts. These cognitive processes can be effectively studied in ensemble musicians according to the proposed "design taxonomy" by manipulating - systematically and parametrically - the degree of musical interaction. This plurality in approach and 
methods could potentially support the investigation of the basic building blocks of nonverbal communication and social cognition, which are human capacities that likely provided the foundations from which music itself evolved [12]. The use of music as a model of social interaction has the potential to disclose how these early building blocks were assembled in the course of human phylogeny.

Novel experimental observations, questions and theories are likely to emerge from this approach and impact upon cognitive science on a broader scale. Musical ensembles thus constitute a promising experimental platform for implementing ecological and fully interactive scenarios that capture the richness and complexity of human social interaction. The features of musical ensemble performance and a taxonomic approach to degrees of musical interaction provide valuable tools for pursuing a new line of cognitive neuroscience research. 


\section{References}

[1] Wing, A.M. et al. (2014) Optimal feedback correction in string quartet synchronization. J. R. Soc. Interface 11(93), 20131125.

[2] D'Ausilio, A. et al. (2012) Leadership in orchestra emerges from the causal relationships of movement kinematics. PLoS One 7(5), e35757.

[3] Badino, L. et al, (2014) Sensorimotor communication in professional quartets. Neuropsychologia 55(1), 98-104.

[4] Glowinski, D. et al., (2013) The movements made by performers in a skilled quartet: a distinctive pattern, and the function that it serves. Front. Psychol. 4, 841.

[5] Novembre, G. et al. (2012) Distinguishing self and other in joint action. Evidence from a musical paradigm. Cereb. Cortex. 22(12), 2894-903.

[6] Novembre, G. et al., (2014) Motor simulation and the coordination of self and other in real-time joint action. Soc. Cogn. Affect. Neurosci. 9 (8): 1062-1068

[7] Sänger, J. et al., (2013) Directionality in hyperbrain networks discriminates between leaders and followers in guitar duets. Front. Hum. Neurosci. 7, 234.

[8] Sänger, J. et al., (2012) Intra- and interbrain synchronization and network properties when playing guitar in duets. Front. Hum. Neurosci. 6, 312.

[9] Babiloni, C. et al., (2011) Simultaneous recording of electroencephalographic data in musicians playing in ensemble. Cortex 47(9), 1082-90.

[10] Loehr, J.D. et al., (2013) Monitoring individual and joint action outcomes in duet music performance. J. Cogn. Neurosci. 25(7), 1049-61.

[11] Kokal, I. et al., (2011) Synchronized drumming enhances activity in the caudate and facilitates prosocial commitment--if the rhythm comes easily. PLoS One 6(11), e27272.

[12] Kirschner, S. and Tomasello, M. (2010) Joint music making promotes prosocial behavior in 4-year-old children. Evol. Hum. Behav. 31(5), 354-364.

[13] Fairhurst, M.T. et al., (2013) Being and feeling in sync with an adaptive virtual partner: brain mechanisms underlying dynamic cooperativity. Cereb. Cortex 23(11), 2592-600.

[14] Fairhurst, M.T. et al., (2014) Leading the follower: an fMRI investigation of dynamic cooperativity and leader-follower strategies in synchronization with an adaptive virtual partner. Neuroimage 84, 688-97. 
[15] Ragert, M. et al. (2013) Knowing too little or too much: the effects of familiarity with a co-performer's part on interpersonal coordination in musical ensembles. Front. Psychol. 4, 368. 


\section{BOX 1: Features that make music a promising avenue for social cognition research}

1 - Ecological validity: ensemble musicians participate in a socially relevant interaction, obviating the need to introduce an artificial task, manipulation, or training to induce a social context.

2 - Motivational factors: motivation is an inherent part of music and hence it is not necessary to employ extrinsic techniques (monetary compensation, competition, or response-contingent reward) to trigger the emergence of interaction.

3 - Generalizability: musicality is a widespread human capacity, enabling almost everyone to sing together with others and to produce rhythms through body movements (e.g., simple drumming or dance).

4 - Multi-level interactivity: information transfer is both continuous (body movements) and discrete (musical sounds). Furthermore, musicians' movements that function to produce sound on an instrument can be dissociated from those that are not necessary for sound production (e.g. ancillary movements that serve expressive functions) [15]. These properties allow the investigation of the multi-level communicative functions (hierarchical musical structure and expressive intentions) of musical social interaction.

5 - Temporal dependencies: information transfer is not only based on the content of an individual's instantaneous response but also is affected by rhythmic timing, tempo, and the degree of interpersonal synchrony.

6- Formal description of interaction: the musical score is a script-like description of the interaction that the experimenter can manipulate to control the emergence of social structures and different roles (e.g. leader vs. follower) played by each musician.

\section{Glossary}

Corticospinal excitability: Corticospinal excitability quantifies the efficacy of transsynaptic corticospinal excitation, which is based on the strength of the descending cortical input and the local excitability of the spinal motoneurons. It provides a measure of neural motor activation and is thus often taken as a direct index of simulation processes running in the motor system.

rTMS: Repetitive transcranial magnetic stimulation is used to induce reversible modulations of cortical excitability. rTMS can thus be employed to investigate the causal contribution of a specific brain area to a given cognitive process by inducing virtual brain lesions. 
Sensorimotor synchronization: The temporal alignment of rhythmic movements with external rhythmic events. Ensemble musicians synchronize the sounds that they produce and the expressive body movements that accompany their performances in order to fulfill aesthetic, communicative, and social goals.

Hyperscanning: Neuroimaging methods involving the simultaneous recording of multiple individuals' brain activity. Hyperscanning allows the brain responses from multiple subjects - and their interrelationship - to be quantified during social interaction.

Granger causality: An auto-regressive statistical method for determining whether two time series are causally related to one another. The method involves estimating the ability of past values from one time series to predict future values of another time series.

Intra-brain information flow: Patterns of coherent neural oscillations across distinct anatomical regions within an individual's brain reflect network-wide neural computations. The emergence of neuronal coherence is often assumed to indicate that information is being transferred within a selected sub-population of computational units.

Inter-brain information flow: Synchronous brain activity in two (or more) interacting individuals can arise through mutual modulations of neural activity. Such inter-brain synchronization may represent the information transfer between participants and thereby support interpersonal action coordination. 


\section{Acknowledgements}

We wish to thank Maestro Riccardo Muti for the precious insight he provided us about the fabric of sensorimotor conversation in orchestras. We also wish to acknowledge the valuable collaboration of the Casa Paganini group in Genova, led by Prof. Antonio Camurri. This work has been supported by European Union FP7 grants SIEMPRE, POETICON++ and EBRAMUS, in addition to the MARCS Institute (University of Western Sydney) and the Max Planck Society. 


\section{Figure 1: Experimental design taxonomy of musical interaction}

Schematic illustration of a taxonomy of experimental designs for studying musical interaction. The degree of ecological interaction increases, from left to right, while experimental control decreases. Moving along the continuum shows how the potential for inter-individual behavioral coupling (unidirectional to multidirectional) is augmented while uncertainty (low to high) concerning others' actions increases.

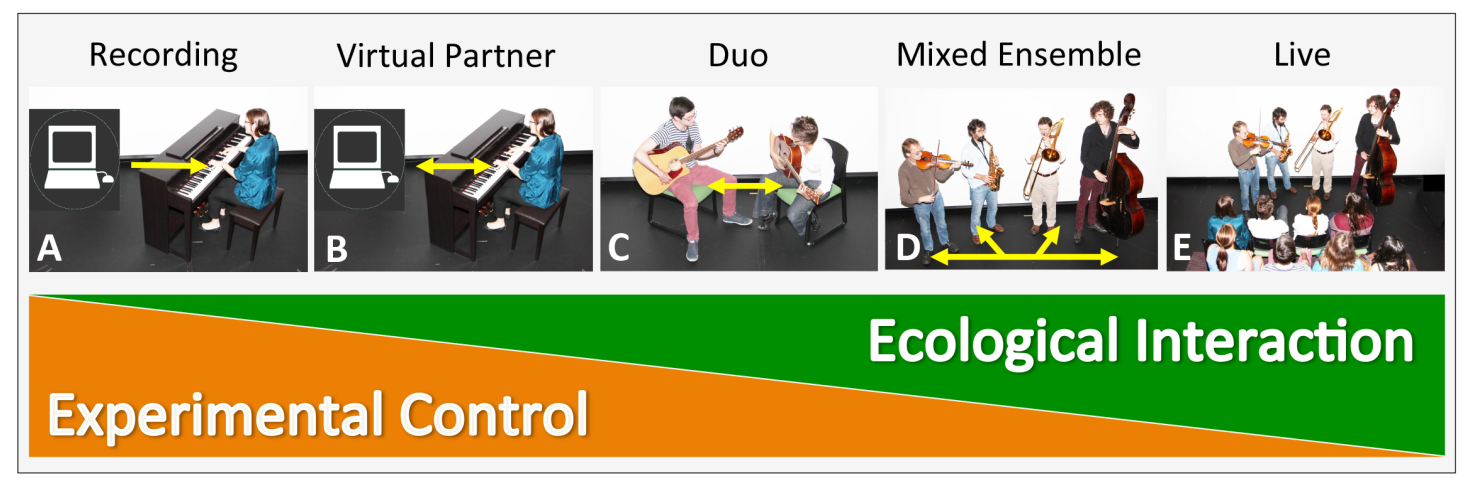

